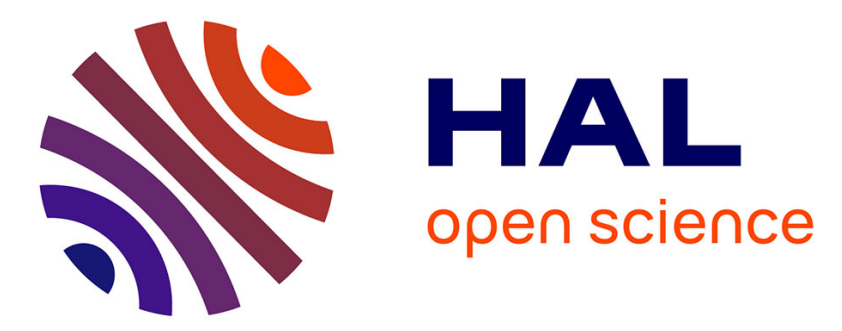

\title{
Facteurs psychosomatiques intervenant dans le développement de l'enfant
}

\author{
J.M. Hascoët
}

\section{To cite this version:}

J.M. Hascoët. Facteurs psychosomatiques intervenant dans le développement de l'enfant. Bulletin de l'Académie Nationale de Médecine, 2021, 10.1016/j.banm.2021.03.008 . hal-03265166

\section{HAL Id: hal-03265166 https://hal.science/hal-03265166}

Submitted on 19 Jun 2021

HAL is a multi-disciplinary open access archive for the deposit and dissemination of scientific research documents, whether they are published or not. The documents may come from teaching and research institutions in France or abroad, or from public or private research centers.
L'archive ouverte pluridisciplinaire HAL, est destinée au dépôt et à la diffusion de documents scientifiques de niveau recherche, publiés ou non, émanant des établissements d'enseignement et de recherche français ou étrangers, des laboratoires publics ou privés. 
Séance thématique : Devenir de l'enfant et neurodéveloppement

Facteurs psychosomatiques intervenant dans le développement de l'enfant

MoTs-Clés : enfant, trajectoires neurodéveloppementales ; interventions socio-éducatives ; résilience.

Psychosomatic factors involved in infant development

KEY-WORDS: child; neurodevelopmental trajectories; socio-educative interventions; resilience.

\section{Jean-Michel HASCOËT}

Université de Lorraine, DevAH EA3450, Vandoeuvre 54500 et Service de Néonatologie,

Maternité Régionale Universitaire A. Pinard, CHRU Nancy, 10 rue du Dr Heydenreich, 54035 NANCY

Courriel : jean-michel.hascoet@ univ-lorraine.fr 


\title{
Résumé
}

Dès la naissance, la qualité de la relation parents enfant influence son développement psychosomatique. Des travaux en neurobiologie suggèrent l'existence d'une relation étroite entre les systèmes de réponse au stress, ocytocine et corticostéroïdes notamment, et l'adaptation à la maternalité. Chez l'enfant, la trajectoire développementale qui suit la naissance comporte des étapes de vulnérabilité qui sont autant d'opportunités d'intervention pour mettre l'enfant dans une situation lui permettant d'exprimer toutes ses potentialités. Des études d'interventions précoces à 4 mois, 12 mois et à l'âge scolaire montrent ainsi une efficacité en termes de résilience des enfants. Elles permettent de limiter l'échec scolaire et sociétal, du fait d'une trajectoire neurodéveloppementale perturbée.

\begin{abstract}
From birth, the quality of the parent-child relationship influences their psychosomatic development. Works in neurobiology suggest the existence of a close relationship between stress response systems, oxytocin and corticosteroids in particular, and adaptation to motherhood. In children, the developmental trajectory following birth includes stages of vulnerability which are all opportunities for intervention to place the child in a situation that allows him to express all his potential. Early intervention studies at 4 months, 12 months and at school age show effectiveness in terms of children's resilience. They make it possible to limit academic and societal failure, due to a disrupted neurodevelopmental trajectory.
\end{abstract}


Le développement psychosomatique et neurologique de l'enfant suit une trajectoire non linéaire, dont l'orientation initiale, définie par l'établissement du lien mère-enfant et parentsenfant dès la naissance, est suivie par de nombreux virages liés à l'environnement. Ceux-ci peuvent être bénéfiques (virages positifs ou résilients) ou néfastes (virages négatifs ou fragilisants) [1]. Ces virages représentent des chances ou des aléas tels que décrits dans l'article de Paul Vert. Ils peuvent avoir des conséquences épigénétiques avec des effets transgénérationnels; ils représentent des opportunités d'intervention à des âges de vulnérabilité qui peuvent être anatomiques et fonctionnels tels que décrits dans l'article de Pierre Gressens, ou liés à l'environnement [1] comme l'a signalé Mario Speranza dans son manuscrit.

Les études chez le rongeur confirment le lien entre le développement anatomique et fonctionnel du système nerveux et le développement du lien parent-enfants. Ainsi, l'ocytocine, sécrétée à partir des noyaux paraventriculaire et supra-optique de l'hypothalamus et qui entraine les contractions utérines lors de l'accouchement et de la délivrance, ainsi que l'éjection du lait lors de l'allaitement, est très fortement associée aux mécanismes d'attachement parent-enfant [2,3]. Dans une méta analyse sur le lien entre les interactions précoces parents-enfant et l'ocytocine, Scatliffe et coll [2] ont trouvé 17 études concordantes montrant que le contact direct parents-enfant, en peau à peau ou lors de l'allaitement par exemple, entraînait une augmentation significative des taux d'ocytocine. Réciproquement, l'expression de récepteurs à ocytocine OXTR CD38, mis en évidence dans des cerveaux de souris, est d'autant plus élevée que la maternalité est importante [4]. Dans une étude chez des souris knockout, les animaux manquant d'ocytocine ne s'occupaient pas de leurs petits et refusaient de les nourrir. Un comportement maternant normal était rétabli par l'administration d'ocytocine [5]. Chez l'humain, dans un travail incluant 352 mères et pères, mesurant les taux sanguins d'ocytocine, les parents avec les taux les plus élevés avaient un comportement plus 
maternant comprenant un contact peau à peau, un toucher des enfants et des périodes de synchronisation du regard parents-enfants significativement plus longs [4]. Par ailleurs, une dualité des corticostéroïdes au plan de l'attachement a été suggérée [6]. Ainsi, les taux de stéroïdes semblent s'élever lors d'un contact affectif positif, sauf s'ils sont préalablement déjà élevés du fait d'un stress antérieur. Cette dualité qui repose en partie sur un feedback négatif de l'axe hypothalamo- hypophyso-surrénalien, serait un facteur majeur d'adaptation à la parentalité [6,7] et de difficultés d'attachement en cas de stress. Dans une revue de la littérature sur l'effet d'un stress précoce, Vanessa Lux suggère que les corticostéroïdes auraient un rôle de médiateur central sur la programmation épigénétique définissant l'orientation du développement des individus. Ce mécanisme expliquerait en partie les effets à long terme d'un stress précoce de la petite enfance [6]. Enfin, les phéromones d'apaisement secrétées par les aréoles mammaires favoriseraient la première intervention positive qu'est l'allaitement maternel sur le développement [7-9].

Lors de l'évolution de l'enfant, il est possible de prévenir l'influence néfaste de l'environnement par des interventions d'autant plus efficaces qu'elles sont proposées lors de fenêtres de vulnérabilité développementales, impulsant ainsi une dynamique positive telle que décrite dans l'article de Catherine Barthélémy. Les interventions précoces par l'éducation des familles à la parentalité, les visites et soutien à domicile ou la facilitation de l'accès aux soins permettent d'établir ou de renforcer un lien sécurisant dès l'âge de 3-4 mois, âge de reprise du travail maternel, et autour de 12 mois, à l'âge de la verticalisation et de l'autonomisation par la marche. Il est intéressant de noter que les interventions sont d'autant plus efficaces que les 2 parents sont impliqués. [10]

Lors de l'entrée dans le système scolaire, du fait de l'importance des apprentissages fondamentaux, vers 6-7 ans, la prise en compte de la relation entre la mobilité, parfois excessive et catégorisée «hyperactivité », et les fonctions attentionnelles, ouvre des 
perspectives intéressantes. En effet, plusieurs études suggèrent que la mise en mouvement du corps pourrait correspondre à une recherche inconsciente d'optimisation du niveau d'éveil. Dans une étude évaluant l'effet de la mobilité corporelle sur les fonctions attentionnelles chez des enfants vulnérables nés prématurément, par rapport à des témoins de même âge nés à terme, nous avons montré qu'à 6 ans leur control postural était encore immature. Leur donner la possibilité d'augmenter leur mobilité et les libérer de consignes contraignantes comme celle de rester assis sans bouger, comme à l'école, a entraîné une amélioration de leurs fonctions attentionnelles, en particulier l'attention et la résistance à la distraction. [11,12]. Dans une étude de 387 enfants adressés à l'âge de 6 ans pour hyperactivité avec déficit d'attention, Krasner et coll. ont montré qu'une prise en charge adaptée, permettait d'observer une résilience à l'âge de 9 ans chez 44\% d'entre eux [13]. Parmi ceux qui présentaient encore des difficultés cognitives et comportementales à cet âge, 39\% avaient récupéré des capacités normales à l'âge de 16 ans [13]. Toutefois, ces enfants, malgré l'amendement de leurs difficultés attentionnelles, ne parviennent pas à compenser les retards accumulés en plus des nouvelles connaissances à acquérir. Ainsi, les interventions socio-éducatives précoces, et renouvelées à chaque étape clé du développement, prennent-elles une dimension de santé publique. Elles permettent de créer les conditions favorables pour que les enfants puissent exprimer tout leur potentiel, évitant une accumulation des retards qui impactent leur devenir en les conduisant à l'échec scolaire et sociétal, du fait d'une trajectoire neurodéveloppementale perturbée.

\section{Déclaration publique d'intérêt :}

L'auteur déclare ne pas avoir de liens d'intérêt en relation avec le contenu de cet article 


\section{REFERENCES}

1. Ho S-M, Johnson A, Tarapore P, Janakiram V, Zhang X, Leung Y-K. Environmental Epigenetics and Its Implication on Disease Risk and Health Outcomes. ILAR J. 2012;53(3-4):289- 305.

2. Scatliffe N, Casavant S, Vittner D, Cong X. Oxytocin and early parent-infant interactions: A systematic review. Int J Nurs Sci. 2019;6(4):445- 53.

3. Leckman JF, Feldman R, Swain JE, Eicher V, Thompson N, Mayes LC. Primary parental preoccupation: circuits, genes, and the crucial role of the environment. J Neural Transm [Internet]. juill 2004 [cité 8 févr 2021];111(7). Disponible sur: http://link.springer.com/10.1007/s00702-003-0067-x

4. Feldman R, Zagoory-Sharon O, Weisman O, Schneiderman I, Gordon I, Maoz R, et al. Sensitive Parenting Is Associated with Plasma Oxytocin and Polymorphisms in the OXTR and CD38 Genes. Biol Psychiatry. 2012;72(3):175- 81.

5. Yamamoto Y, Liang M, Munesue S, Deguchi K, Harashima A, Furuhara K, et al. Vascular RAGE transports oxytocin into the brain to elicit its maternal bonding behaviour in mice. Commun Biol. 2019;2(1):76.

6. Lux V. Epigenetic Programming Effects of Early Life Stress: A Dual-Activation Hypothesis. Curr Genomics. 2018;19(8):638- 52.

7. Lester BM, Conradt E, LaGasse LL, Tronick EZ, Padbury JF, Marsit CJ. Epigenetic Programming by Maternal Behavior in the Human Infant. Pediatrics. 2018;142(4).

8. Schaal B, Doucet S, Sagot P, Hertling E, Soussignan R. Human breast areolae as scent organs: Morphological data and possible involvement in maternal-neonatal coadaptation. Dev Psychobiol. 2006;48(2):100- 10.

9. Kaba H, Fujita H, Agatsuma T, Matsunami H. Maternally inherited peptides as strainspecific chemosignals. Proc Natl Acad Sci. 2020;117(48):30738- 43.

10. Mountain G, Cahill J, Thorpe H. Sensitivity and attachment interventions in early childhood: A systematic review and meta-analysis. Infant Behav Dev. 2017;46:14- 32.

11. Hascoët JM, J Rosenbaum, I Hamon, H Ceyte La mobilité corporelle influence les capacités d'inhibition de l'enfant de 7 ans né prématuré : réflexions préliminaires. Bull Acad Natl Méd 2019;203; 598-602

12. Rosenbaum J, Ceyte H, Hamon I, Hascoët AMJ, Petel A, Caudron S, Hascoët JM. Influence of prematurity and mobility on the three attention networks at school age. Philadelphie E-PAS2020; 2275.2

13. Krasner AJ, Turner JB, Feldman JF, Silberman AE, Fisher PW, Workman CC, et al. ADHD Symptoms in a Non-Referred Low Birthweight/Preterm Cohort: Longitudinal Profiles, Outcomes, and Associated Features. J Atten Disord. 2018;22(9):827- 38. 